\section{Modeling and Analysis of WAP protocol Familyl}

\author{
Marina Alekseeva, Ekaterina Dashkova, \\ Dmitry Chaly \\ P.G. Demidov Yaroslavl State University \\ 150000 Yaroslavl, Sovetskaya 14, Russia \\ marya_87@mail.ru, dea.yar@mail.ru, \\ chaly@uniyar.ac.ru
}

\begin{abstract}
The importance of data networks and multimedia cannot be overestimated in contemporary world, at the dawn of Information Era. Information collection, processing and distribution systems are the key points for applying the scientific knowledge. Specification and verification of communication protocols and boosting their performance became the topics of the day. Due to the development of mobile systems the wireless networks are in good demand.

Thanks to technological advances in wireless data transfer the hardware and software developers offer a wide range of new services like mobile internet. Innovations must be useful, convenient, flexible, fast and secure enough with the least possible amount of errors during operation.

One of the ways to provide mobile internet is a WAP stack of protocols. WAP abbreviation translates into Wireless Application Protocol or Wireless Access Protocol. The second reading is more precise in reflecting the meaning of WAP technology, purpose of which is to grant access to information stored directly in the Internet.

We propose a modification for Wireless Transaction Protocol (WTP is the transport layer of WAP) which improves the original flow control algorithm. The work includes new ideas of developing and improving WAP as one of the important technologies.We use NS2 (Network Simulator) which provides an opportunity to specify such network protocols and simulate their behaviors.

Index terms: Wireless Application Protocol, Network Simulator, flow control protocol

\section{INTRODUCTION}

In the beginning of the 21st century computer networks became the most important way of communication. Wireless networks spread worldwide and became one of the contemporary technologies. There are several protocols which provide access to the wireless internet. One of the most popular of them at the beginning of the 21st century was Wireless Access Protocol (WAP) [6]. The most exact definition of WAP technology: to provide access to the information from the internet for the mobile devices which have some specific characteristics:

- amount of memory devices;

- small size of the phone's screen, as well as the limitations of his keyboard;

- low processor speed;

- low bandwidth of the communication channel;

- possible long timeouts.

WAP was developed to overcome these problems and that is its major difference from HTTP and TCP/IP. Note that the user does not resort to the assistance of additional devices, such as modem. WAP is a protocol that describes the way in which information from the Internet displays on the small screen of mobile phone.

WAP is a stack of protocols which consists of several layers. According the OSI (Open Systems Interconnection) model [8] WAP contains six layers (Application layer, Session layer, Transaction layer, Secure layer, Wireless Datagram Protocol and Bearers). Each layer is intended to perform a welldefined function. We are focusing on the transport layer. In our paper we are focusing on the transport layer, modifying flow control algorithm using ideas, which are the fundamental principles of ARTCP algorithm [1].This protocol offers a new method of flow control based on rate control of the transfer of segments in the network.

Protocol ARTCP (Adaptive Rate Transmission Control Protocol) borrows some of the mechanisms of the TCP protocol which is the main transport protocol of the internet. Protocol ARTCP differs from TCP standard. Segments are sent to the network not in the form of the burst, but separated by time intervals. Sending rate depends on receiving rate as both parameters are included into the ratio which is changing the principle of system's work. Algorithm 
has a mechanism of adaptating according to the evolving conditions of a network.

We will check properties of our algorithm with the help of a network simulator. There are two modeling approaches: analytical approach and simulation approach. At the moment with the help of NS2, which provides an opportunity to specify such network protocols and simulate their behaviors, our model of the modified protocol WTP is in its final step of development.

\section{MAIN PART}

\section{A. System modeling. NS2.}

Simulation is widely-used in system modeling for applications ranging from engineering research, business analysis, manufacturing planning, and biological science experimentation [5].

In the development of such kind of model we can use a static models as well as dynamic ones. And under the static model we understand the models which can be used to analyze the network conditions in the particular curtain moments of time, for example, analytical methods of calculations from the queuing theory. Under the dynamic model we understand - discrete stochastic models, for example, processes of generation of requests or processes of their services. A great amount of programming features for imitating modeling exists nowadays: libraries of functions for the standard compilers as well as specific programming languages [7].

There are some examples which show that different performance indicators can be took off from the model with the help of colored Petri Nets. CPN/Tools - is an instrument that gives an opportunity of visualized corrections, performance and analysis of colored Petri Nets (CPN - Colored Petri Nets). In $\mathrm{CPN}$ /Tools non classical variant of colored Petri Nets are used: time is added in the structure as well as embedded programming language is used CPN ML (it is based on Standard ML). That is why Network Simulator is the most perspective specialized packet for different performance characteristic analysis of various of protocols. NS2 allows to build communication protocol models of almost all kinds of complexity. There exist some protocol models which were built by the protocols developers with the help of NS2. This fact can tells much about the high quality of the models developed with this instrument $[2]$.

Network Simulator (version 2), widely known as $\mathrm{NS} 2$, is an event driven simulation tool which is very useful in studying the dynamic nature of communication networks. NS2 provides users with a way of specifying such network protocols and simulating their behaviors.

NS2 allows to create different types of traffic from the simplest ones which obeys the Poisson law to self-similar. Undoubted advantage of this simulator for the wireless network situation is its ability to define the nodes movements with the help of Scenario Generator mechanism. It is important to underline embedded opportunity of animation of different scenario results. Full as well as mere version of NS2 contains an instrument for animation of results of the model performance - nam (Network Animator). It is implement on Tcl/Tk. Only full version of NS2 contains a tool Xgraph. It helps graphically display the modeling results. This fact is very important as its significantly simplify efforts for results visualizing (there is no need to install specific software especially for providing visibility of model performance).

NS2 suggest two steps of work. The first step is constructing a model with the help of programming on $\mathrm{C}++$, and finally the use of the Object-oriented Tool Command Language ( $\mathrm{OTcl}$ ) for analysis of the model and simulating the network conditions. It allows us to include our $\mathrm{C}++$ programming code to the NS2 environment (fig.1) [3].

We decided that NS2 is the most convenient tool for modeling the network behavior.

B. WTP.

The Wireless Transaction Protocol is responsible for reliable message delivery. Maximum Transfer Unit (MTU) is a maximum size of a packet in networks. WTP fragment and reassembly the messages that exceed the size of the network MTU (Maximum Transfer Unit). There are three classes of operation for this protocol. We are focusing only on class-2 operation for Wireless Transaction Protocol. Flow control in cases of fragmented messages, is performed by sending fragments in groups. Every group of 


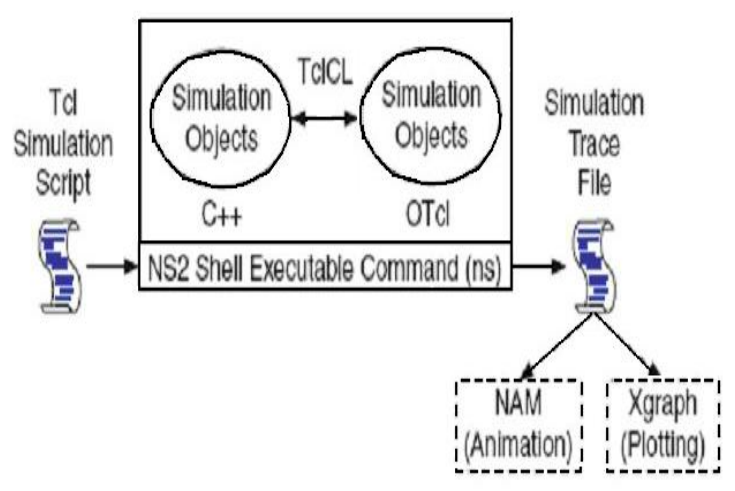

Рис. 1: Basic architecture of NS2

packets requires only one acknowledgement. The last packet of each group contains a special flag. This flag indicates the end of the group and receiver knows when to send an acknowledgment. The size of each group depends on the link characteristics and the device memory. It is necessary to avoid extra packet retransmission and data loss. Receiver sends a negative acknowledgement (NAK) if the end-of-group packet is received whilst intermediate packets are missing. This operation is repeated until the entire group is received and a positive acknowledgment is sent. If timeout expiries before any acknowledgement is received $(\mathrm{ACK} / \mathrm{NAK})$, then only the last packet of the group is retransmitted, and sender knows what packets have been lost. Wireless Transaction Protocol tries to minimize the number of unnecessary retransmissions [4].

\section{Proposed model.}

Extended Segmentation and Re-assembly of WTP protocol use either a sliding window based transmission or the traditional stop and wait mechanism [6]. The fundamental ideas of sliding window and stop and wait protocols are classical. They are used in various of transport protocols, but we presumes that this algorithms can be improved or more effective analogue can be developed. ARTCP flow control algorithm is based on the sliding window algorithm as well as on its own mechanism. The major advantage of ARTCP algorithm is that fact that it doesn't interpret the packet loss just as network overflow, this fact helps to avoid undesirable decrease of efficiency of network performance.

In our model we have three parameters, which we will change depending on network performance. Let $t_{s}$ is the time interval between consecutive packets of the group which are sent from the sender SENDER to the receiver RECEIVER. And $t_{r}$ is the interval between consecutive packets of the group which are received by the RECEIVER.

$$
t_{r}=t_{r-1} * a l f a+(1-a l f a) * t^{\prime},
$$

where

$$
\text { alf } a=\frac{1}{\left(A m_{p}-1\right)},
$$

$t^{\prime}=\left(\right.$ now $\left.-t_{r l}\right)$, now - current time, $t_{r l}$ - the time when the previous packet of the group was received. Let $A m_{p}$ - as the number of packets in the group. In our model there are two types of acknowledgments (ACK is a positive acknowledgment, NAK is a negative acknowledgment) (fig.2). When receiver sends an acknowledgment it transfers $t_{r}$ with the help of it. Sender calculates the ratio $\frac{t_{s}}{t_{r}}$. Depending on the result of this ratio sender has several situations for analysis and further actions. The first one $\frac{t_{s}}{t_{r}}=1$ reflects the perfect network conditions. The second one is $0.85<\frac{t_{s}}{t_{s}}<1$. All parameters can be changed by increasing $A m_{p}$, decreasing $t_{s}$ and timeout:

$$
A m_{p}=2 * A m_{p} ;
$$

(exponentially increase $A m_{p}$ );

$$
t_{s}=\frac{t_{s}}{2}
$$

(exponentially decrease $t_{s}$ );

$$
\text { timeout }=\frac{\text { timeout }}{2}
$$

(exponentially decrease timeout);

We conclude that if $0.70<\frac{t_{s}}{t_{s}}<0.85$ there is no enough data for our algorithm to make a decision how to modify parameters (conditions of a network correspond to the established parameters). The fourth one is $\frac{t_{s}}{t_{r}}<0.70$, so the network is congested, all parameters can be changed by decreasing $A m_{p}$, increasing $t_{s}$ and timeout: 


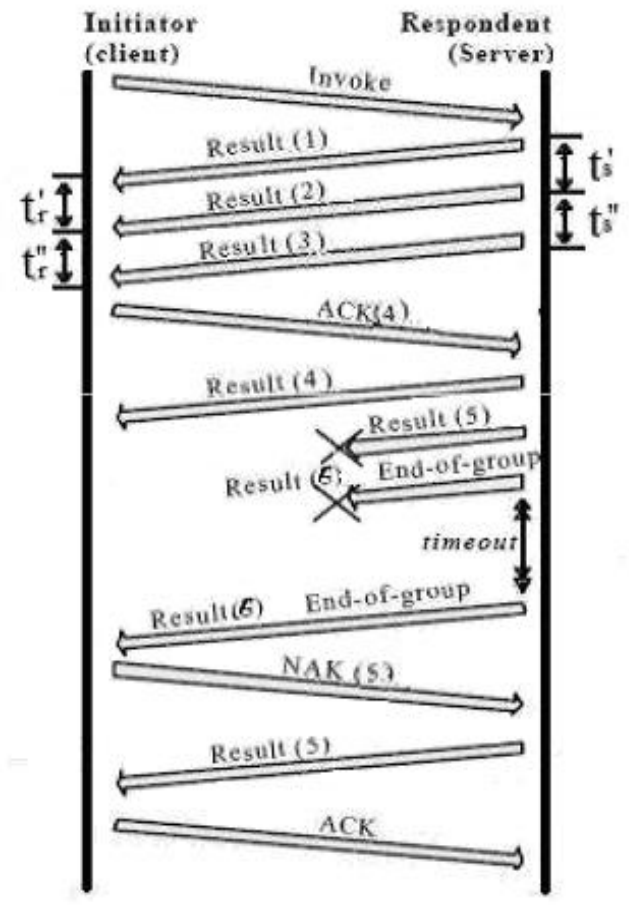

Рис. 2: Scheme of work

$$
A m_{p}=\frac{A m_{p}}{2}
$$

(exponentially decrease $A m_{p}$ );

$$
t_{s}=2 * t_{s}
$$

(exponentially increase $t_{s}$ );

$$
\text { timeout }=2 * \text { timeout }
$$

(exponentially increase timeout);

\section{CONCLUSION}

With the growth and development of communication systems the economic benefit from increased efficiency of communication protocols (such as WAP) can be very substantial. WAP requires serious improvement. We have developed a new method of flow control, which is based on the management of transmission speed, the number of outgoing information and the waiting time for a response from the recipient.

We concluded that the control algorithms of data streams using the insufficient number of network features. So they faced with the same error: overloading the network, and the subsequent loss or corruption of data. We propose the idea of the analysis of additional characteristics of the network and its subsequent improvement, depending on the incoming data.

We analyzed the transport layer (Wireless Transaction Protocol - WTP) of WAP protocol and concluded that the mechanism of flow control can be flexible and adapt to the conditions of the wireless environment. Wireless channels are characterized by high levels of errors and narrow bandwidth, therefore the network congestion occurs frequently (duration of waiting for a response from the server increases or data is lost). We have studied the weaknesses of the flow control mechanism of several protocols. We have made changes in the structure of the transmitted packets, entered additional information in the packet header, in a number of key functions of the protocol, which are responsible for sending, receiving packets and settings of timeouts. In the future, we can create a protocol based on verified and analyzed model.

\section{ACKNOWLEDGMENTS}

The article was prepared within the scope of Finnish - Russian University Cooperation in Telecommunications Program. The authors would also like to thank the dean of Yaroslavl Demidov State University Computer Science Department P.G. Parfenov for interest and support of this project and the head of scientific-educational center "Center of Innovation Programming"Professor V.A. Sokolov for helpful advices.

\section{REFERENCES}

[1] I. V. Alekseev, V. A. Sokolov, D.U. Chaly "Modeling and analysis of Transport protocols for computer networks", Yaroslavl State University, 2004. (in Russian) 
[2] V. A. Sokolov, D.U. Chaly, "Methods for investigating the behavior of transport protocols in congested network Distributed Inform.computational resources, and math. modeling, MKVM-2004, p.129.

[3] T. Issariyakul, E. Hossain, "Introduction to Network Simulator NS2", DOI: 10.1007/978-0-38771760-9 1, Springer Science + Business Media, LLC 2009, p.20-23.

[4] C. Ladas, R. M. Edwards AMIEE, G. Manson "WAP performance on an end-to-end scheme", The Centre for Mobile Communications Research (C4MCR), The University of Sheffield.

[5] http://www.isi.edu/nsnam/ns/

[6] http://www.openmobilealliance.org/Technical/ wapindex.aspx - WAP specification.

[7] Y. Koucheryavy "NS-2 as a universal means of simulation networks", Tampere University of Technology, Telecommunications Laboratory, Tampere, Finland.

[8] A.S. Tanenbaum "Computer networks", - St. Petersburg, Peter, 2003. - 992 p.

[9] Aust Stefan, Nikolaus A. Fikouras, Görg Carmelita "Enabling Mobile WAP Gateways using Mobile IP", Department of Communication Networks (ComNets), Center for Information and Communication Technology (IKOM) University of Bremen, Germany.

[10] Moon Il-Young "Performance Analysis of WAP Packet Transmission Time and Optimal Packet Size in Wireless Network", School of Internet Media Engineering, Korea University of Technology and Education, Republic of Korea. Springer-Verlag, Berlin Heidelberg, 2006.

[11] http://nile.wpi.edu/NS/ 